\title{
The Development and Application of High Compression Ratio Methanol Engine ECU
}

\author{
Hong Bin \\ State Key Laboratory of Engines \\ Tianjin University \\ Tianjin, China, 15922184696 \\ hongbinlqyun@163.com \\ Song Feiyu \\ State Key Laboratory of Engines \\ Tianjin University \\ Tianjin, China
}

\author{
Wang Yang \\ State Key Laboratory of Engines \\ Tianjin University \\ Tianjin, China \\ He Yajun \\ State Key Laboratory of Engines \\ Tianjin University \\ Tianjin, China
}

\author{
Song MingZhi \\ State Key Laboratory of Engines \\ Tianjin University \\ Tianjin, China \\ Li Xiaolong \\ State Key Laboratory of Engines \\ Tianjin University \\ Tianjin, China
}

\begin{abstract}
A total design scheme for the high compression ratio methanol engine electronic control unit (ECU) was developed. Chosen the ARM Cortex-M3 based microcontrollers LPC1766 as the main controller of electronic control system, then the signal processing circuit module, main controller system circuit module, actuator driving circuit module were designed. The experiment proves that the hardware are able to satisfy driving and controlling demands of engine ignition and injector solenoid valves, and the whole electronic control system possesses high reliability and antijamming capability as well.
\end{abstract}

Keywords-high compression ratio methanol engine; ECU; hardware; development

\section{INTRODUCTION}

Methanol is a kind of ideal engine alternative fuel, which can reduce harmful engine emissions. High compression ratio spark-ignition methanol engine has characteristics of high compression ratio, airway injection and spark ignition. Its thermal efficiency is close to the diesel engine and the emission performance is better. So it is suitable for large-scale engines. In 2002, the EPA developed a turbocharged, PFI spark-ignited 1.9L, 4-cylinder methanol engine with 19.5:1 compression ratio. The engine operated unthrottled using stoichiometric fueling from full power to near idle conditions, using EGR and intake manifold pressure to modulate engine load. The thermal efficiency of the engine was up to $42 \%$, as high as a diesel engine. The experiment proved the probability of the high compression ratio methanol engine. This paper describes the development of the high compression ratio methanol engine ECU and experiments [1] [2].

\section{THE DESIGN OF ECU}

The target of the hardware design is to meet the high compression ratio methanol engine. So the control accuracy and speed, HCCI, the connection to the upper computer, which is used to debug software and scale data are needed. The ECU includes the following parts: the system control circuit (power supply circuits and communication circuits), the input signal processing circuit (crank signal, cam signal, knock and other analog signal processing circuits) and drive circuits (steppermotor drive circuits, injector drive circuits and oxygen-heating drive circuits).

\section{THE SYSTEM CIRCUITS MODULE}

The structure of the high compression ratio methanol engine ECU is given below in Figure 1.

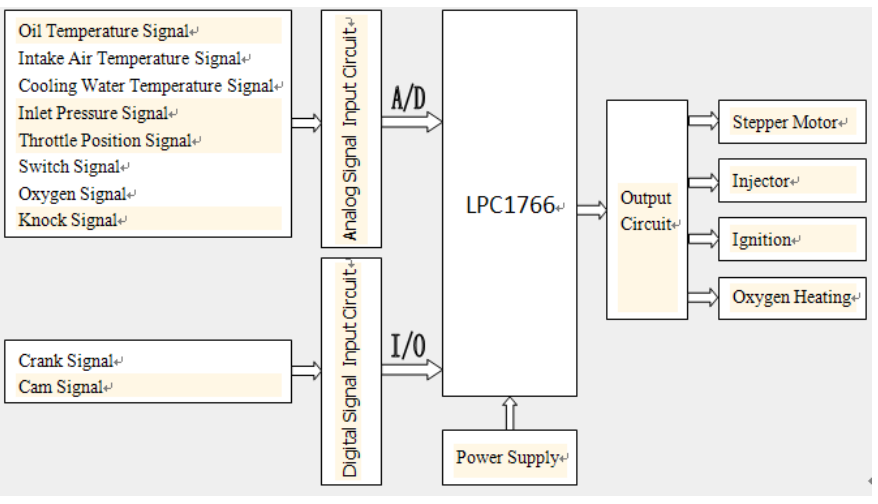

Figure 1. The structure of ECU

\section{A. THE main controller}

The SCM of the ECU in this paper is LPC1766, a 32-bit ARM Cortex-M3 microcontroller. It is used for embedded applications featuring a high level of integration and low power 
consumption. The LPC1766 operates at CPU frequencies of up to $100 \mathrm{MHz}$. It offers high performance and very low power consumption. Its main features are as follows: 1. The ARM Cortex-M3 CPU incorporates a 3-stage pipeline and uses a Harvard architecture with separate local instruction and data buses as well as a third bus for peripherals. The ARM Cortex-M3 $\mathrm{CPU}$ also includes an internal prefetch unit that supports speculative branching. 2. The series of the LPC1700 includes up to $512 \mathrm{kB}$ of flash memory, up to $64 \mathrm{kB}$ of data memory, Ethernet MAC, USB Device/Host/OTG interface, 8-channel general purpose DMA controller, 4 UARTs, 2 CAN channels, 2 SSP controllers, SPI interface, 3 I2C-bus interfaces, 2-input plus 2-output I2S-bus interface, 8-channel 12-bit ADC, 10-bit DAC, motor control PWM, Quadrature Encoder interface, 4 general purpose timers, 6-output general purpose PWM, ultra-low power Real-Time Clock (RTC) with separate battery supply, and up to 70 general purpose $\mathrm{I} / \mathrm{O}$ pins[3].

\section{B. THE SYSTEM CIRCUITS}

The system circuits in this paper mainly include power management module and communication circuit module.

\section{1) POWER MANGEMENT MODULE}

The step-down switching regulator LM2575-5 is used in this paper to provide steady voltage of $5.0 \mathrm{~V}$ to the ECU. The chip has some significant features: guaranteed 1.0A output current, 52 $\mathrm{kHz}$ fixed frequency internal oscillator, thermal shutdown and current limit protection and high efficiency. All the features can develop the efficiency and reliability of the ECU [4].

\section{2) COMMUNICATION CIRCUIT MODULE}

In order to monitor and control the engine running state, modify the control parameters in ECU, bi-directional data exchange between upper computer and ECU is needed. It is realized by RS-232 and CAN. $3 \mathrm{~V}$ to $5.5 \mathrm{~V}$ multichannel RS-232 line driver MAX3232E and high speed CAN-Transceiver are used in this ECU.

\section{INPUT SIGNAL PROCESSING CIRCUIT MODULE}

\section{A. ANGLE SIGNAL PROCESSING CIRCUIT}

There are two kinds of angle signal sensor in this ECU: Electromagnetic sensors (used for the crankshaft position measurement) and Hall sensors (used for camshaft position and speed measurement). The circuits are different due to the different kinds of sensors.

\section{1) HALL SENSOR SIGNAL PROCESSING CIRCUIT}

The voltage of the Hall sensor needed is provided by the ECU power management module. The output of the sensor is $5 \mathrm{~V}$, while the LPC1766 I/O can only accept 3.3V signal maximally. So a transistor is needed to convert $5 \mathrm{~V}$ into $3.3 \mathrm{~V}$. The processing circuits are given below in Figure 2.

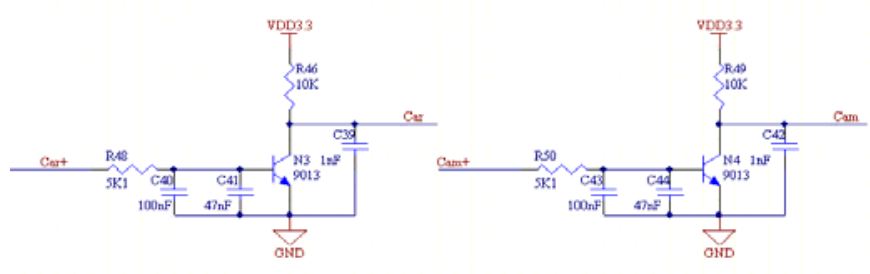

Figure 2. Hall sensor processing circuits

2) ELECTROMAGNETIC SENSOR SIGNAL PROCESSING CIRCUIT

The instantaneous speed of engine is one of the important parameters to judge engine conditions. The accuracy of the measurement has a significant influence on engine performance. So the crankshaft signals should be handled strictly to guaranty its accuracy. The MAX2296UAEE [5] produced by Maxim is used to process the crankshaft signals. The chip which integrates a precision amplifier, comparator with selectableadaptive peak threshold and zero-crossing circuit blocks, precisely matched resistances is suitable for the LPC1766 I/O. It has two differential input channels, so it can process two signals at the same time. The original crankshaft signals and the processed crankshaft signals are given below in Figure 3 and Figure 4. By comparing the two figures, we can clearly see the processed crankshaft signals are more regular.

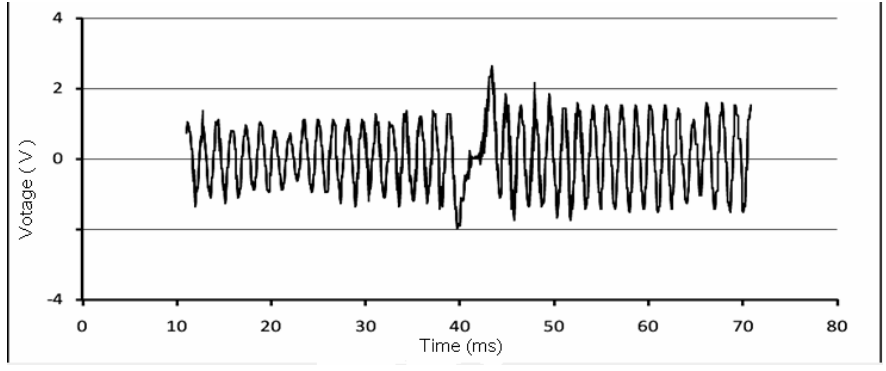

Figure 3. The original crankshaft signals

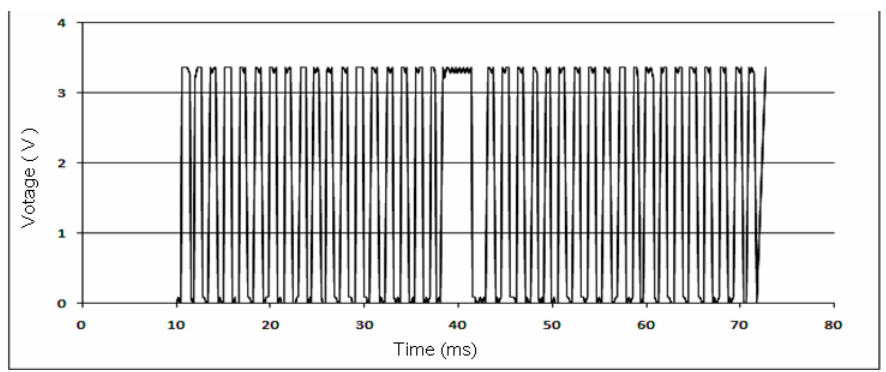

Figure 4. The processed crankshaft signals

\section{B. KONCK SIGNAL PROCESSING CIRCUIT}

The vibration signal exported from knock sensor should be processed by HIP9011 [6], an engine knock signal processor. This chip has some features:1. Amplify the signal. The signal 
from the knock sensor is too small, so it should be amplified before the chip processes it. This adjustment can be made with 64 different gain settings, ranging between 2 and 0.111. 2 . Programmable Bandpass Filter. The filters have a nominal differential gain of 4 . Center frequencies can be programmed from $1.22 \mathrm{kHz}$ to $19.98 \mathrm{kHz}$, in 64 steps. 3. Programmable Integrator. The integrator time constant is software programmable by the Integrator Time Constant. The time constant can be programmed from $40 \mathrm{~s}$ to $600 \mathrm{~s}$, with a total of 32 steps.

The HIP9011 communicates with the SCM via SPI. The knock signal is filtered by external capacitor and resistance firstly. Then it is amplified by amplifiers. Output from these input amplifiers are directed to a channel select mux switch and then into a 3rd order antialiasing filter. The output signal is then directed to two programmable gain stages. The gain stage signals are outputted to two programmable bandpass filter stages. Outputs from the two BPF stages are then full wave rectified before being digitally integrated by the programmable integrator. The integrator output is applied to a line driver for further processing by the ECU. The output of the HIP9011 is an analog signal. As the LPC1766 internal integrates A/D convertor, the signal from the chip can be directly sent to the A/D channel. In a certain time, the voltage is related to the signal, also means whether detonation happens. The knock processing circuit is given below in Figure 5.

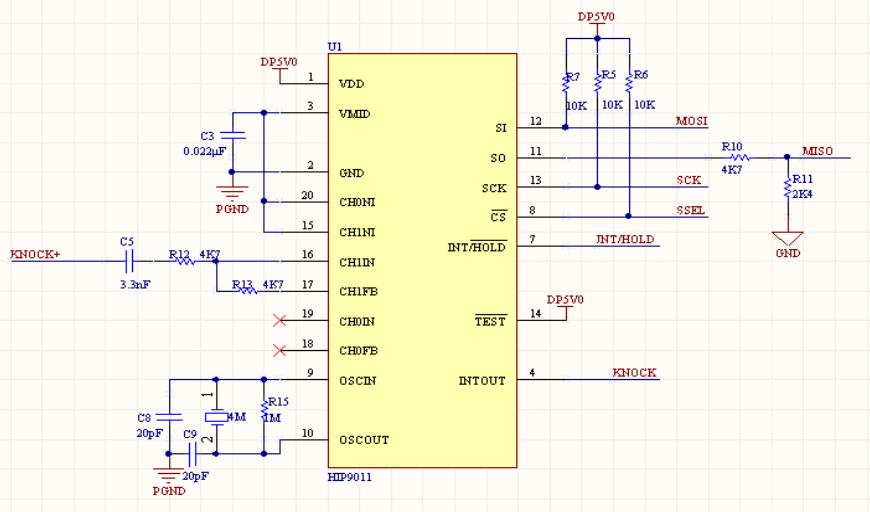

Figure 5. Knock processing circuit

\section{THE OTHER ANALOG SIGNALS PROCESSING CIRCUIT}

The $\pi$ filter is enough for analog signals processing circuits. In order to prevent misconnection to the wiring harness, causing pin damage, a zener diode is parallel connected to the front of each analog signal output. The analog signal processing circuit is given below in Figure 6 .

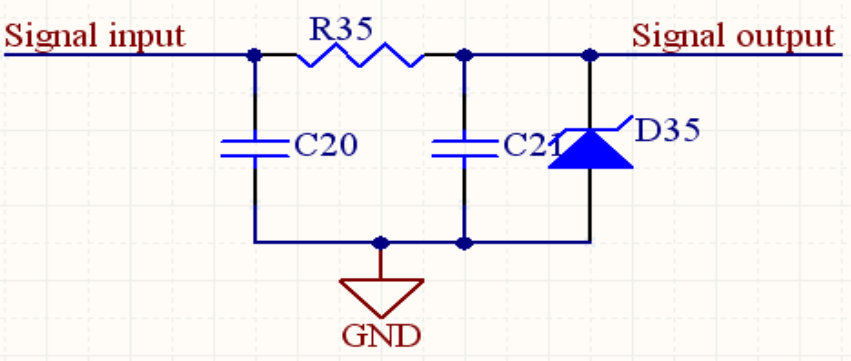

Figure 6. The analog signal processing circuit

\section{THE DRIVE CIRCUIT MODULE}

The ECU drive circuits in this paper include stepper-motor drive circuit, injector drive circuit and oxygen-heating drive circuit.

\section{A. STEPPER-MOTOR DRIVE CIRCUIT}

2-phase stepper-motor driver TLE4729 [7] is used to drive the stepper-motor. The control logic and power output stages for two bipolar windings are integrated on a single chip which permits switched current control of motors with $0.7 \mathrm{~A}$ per phase at operating voltages up to $16 \mathrm{~V}$. The direction and value of current are programmable for each phase via separate control inputs. In the case of low at all four current program inputs the device is switched to inhibit mode automatically. A common oscillator generates the timing for the current control and turn-on with phase offset of the two output stages. The two output stages in full-bridge configuration include fast integrated freewheeling diodes and are free of crossover current. The device can be driven directly by a microprocessor in several modes by programming phase direction and current control of each bridge independently. The application circuit is given below in Figure 7.

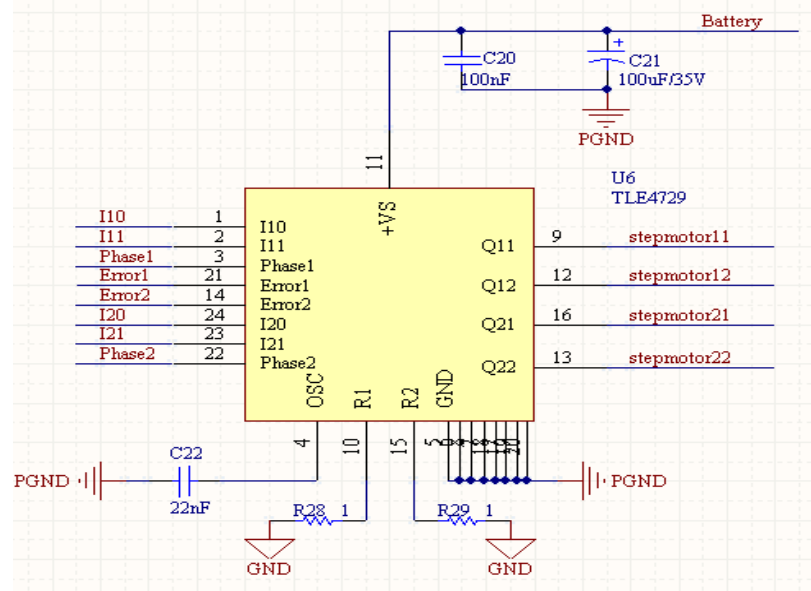

Figure 7. Stepper-motor drive circuit

\section{B. INJECTOR DRIVE CIRCUIT}

Two pieces of TLE6220GP [8] are used to drive six injectors. 
The TLE6220GP is a smart quad low-side switch, which can drive four injectors at the same time. The output current is 1A. The output stages can be controlled direct in parallel for PWM applications (injector coils), or through serial control via the SPI. The application circuits are given below in Figure 8.
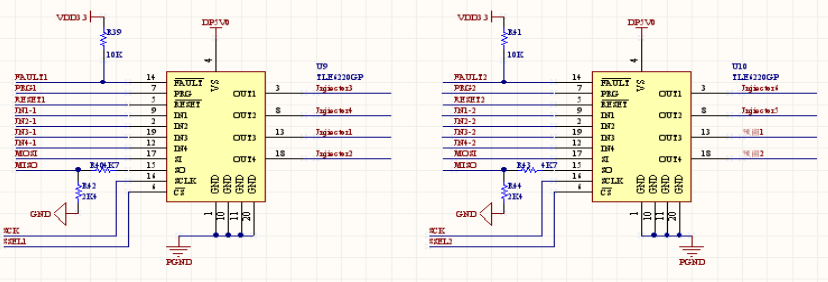

Figure 8. Injector drive circuits

\section{OXYGEN-HEATING DRIVE CIRCUIT}

A piece of BTS5030 [9] is used to drive oxygen-heating module. The BTS5030 is a smart high-side power switch, which can drive two circuits at the same time. The maximum output current is $4 \mathrm{~A}$ and the operating voltage ranges $5 \mathrm{~V}$ to $28 \mathrm{~V}$. The chip also provides protective functions and diagnosis. The application is given below in Figure 9.

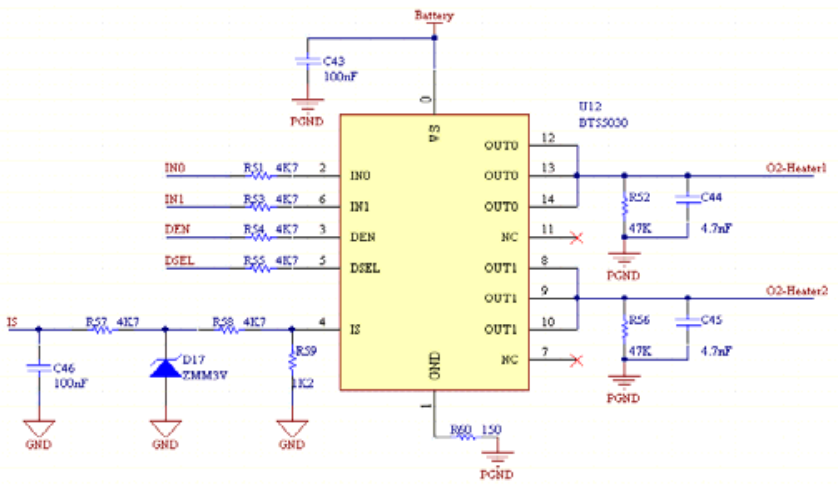

Figure 9. Oxygen-heating drive circuit

\section{THE EMC OF ECU}

The ECU is in a complex electromagnetic environment, ignition interference, actuator electromagnetic interference and other external interference will influence the accuracy of the ECU. In order to eliminate the interference in this paper, some measures have been taken: bold ground to reduce the impedance. Power ground and signal ground are separated to prevent the interaction of two signals. The closed loop ground is also designed. The power modules are close to ground to reduce the impedance. Analog signals are processed through $\pi$ filtration and digital signals are processed through RC filtration. The layout of the ECU is optimized, power chip is as close as the SCM to reduce the power supply system impedance. Crystal is as close as the SCM, and the ground surrounds it to enhance its antiinterference ability. Matching decoupling capacitors are used in every module.

\section{THE EXPERIMENT}

An experiment on high compression ratio methanol engine has been done to test the newly developed ECU. Load characteristics at $1200 \mathrm{r} / \mathrm{min}, 1600 \mathrm{r} / \mathrm{min}, 2000 \mathrm{r} / \mathrm{min}$ and $2400 \mathrm{r} / \mathrm{min}$ are tested. The comparison between the new ECU and the original ECU is given in Figure 10, Figure 11, Figure 12 and Figure 13. From the figures we can see, compared to the original ECU, the newly developed ECU has a better fuel consumption. It means the new ECU has a better performance.

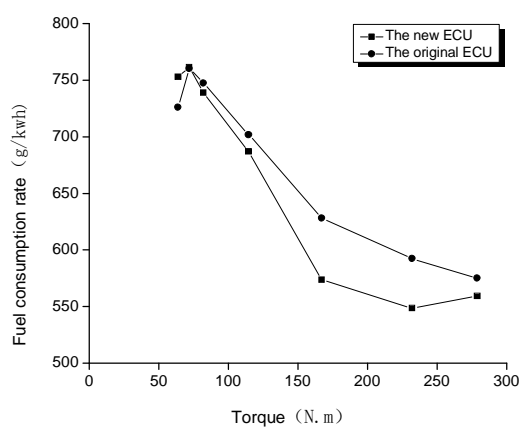

Figure 10. 1200r/min load characteristic

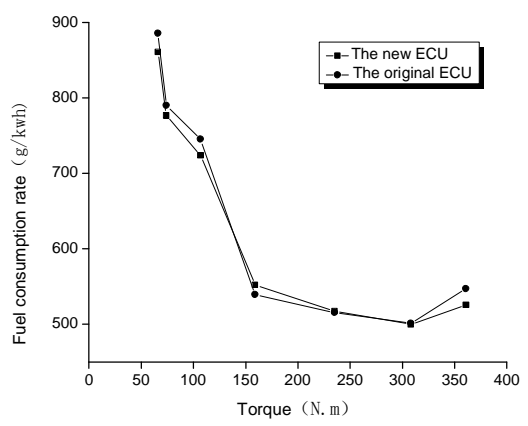

Figure 11. 1600r/min load characteristic

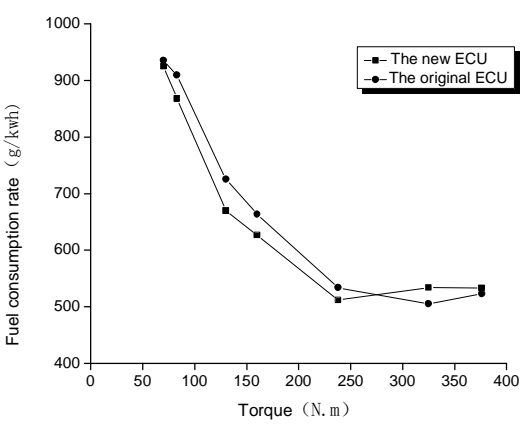

Figure 12. 2000r/min load characteristic 


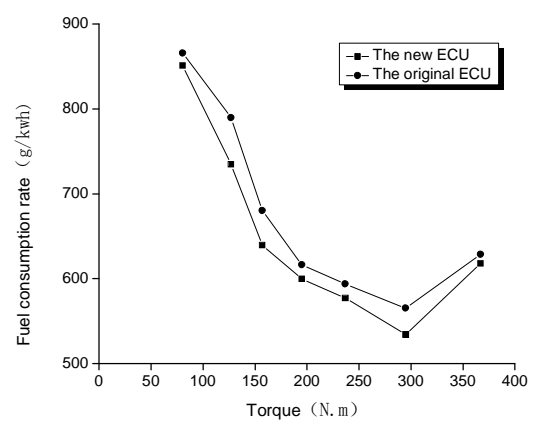

Figure 13. 2400r/min load characteristic

\section{CONCLSUION}

- This paper chooses the LPC1766 to be the master chip of the ECU, describes the system circuit module, signal processing circuit module and actuator drive circuit module of the high compression ratio methanol engine ECU.
- The results of the experiment prove the newly developed ECU mainly reaches the design goal. The new ECU makes the methanol engine work more efficiently.

\section{REFERENCES}

[1] Matthew Brusstar,etal.High Efficiency and Low Emissions from a PortInjected Engine with Neat Alcohol Fuels,SAE Paper 2002-01-2743,2002.

[2] MatthewBrusstar,etal.Economical,High Efficiency engine technologies for Alcohol Fuels,EPA work paper,2002.

[3] Data Sheet LPC1768/66/65/64 .Rev. 02.04. 2008.7.

[4] Data Sheet LM2575/D. Rev.8. 2005.11.

[5] Data Sheet MAX9924-MAX9927. Rev.0. 2008.10.

[6] Data Sheet HIP9011. 1998.11.

[7] 2-Phase Stepper-Motor Driver Bipolar-IC TLE 4729 G.Infineon.2001. 04.

[8] Smart Quad Low-Side Switch Data Sheet TLE 6220 GP V2.1.Infineon.2007.5.

[9] BTS5030-2EKA Data Sheet Rev.2.0,Infineon.2010-08-02. 